\title{
FORMAL PROPERTIES IN SMALL CODIMENSION
}

\author{
JORGE CARAVANTES AND NICOLAS PERRIN
}

\begin{abstract}
In this note we extend connectedness results to formal properties of inverse images under proper maps of Schubert varieties and of the diagonal in products of projective rational homogeneous spaces.
\end{abstract}

\section{INTRODUCTION}

That small codimension subvarieties $Y$ in a sufficently positive variety $X$ inherit some of the properties of $X$ is a well know phenomenon. In this note we study the formal properties of small codimension subvarieties in projective rational homogeneous spaces. Indeed, many of the geometric properties of a subvariety $Y$ in $X$ can be encoded in the algebraic tubular neighbourhood $X_{Y}$ of $Y$ in $X$. In particular if $K\left(X_{Y}\right)$ is the ring of formal functions of $X$ along $Y$ (see Definition 1.1), then, for $X$ normal, the subvariety $Y$ is connected if and only if $K\left(X_{Y}\right)$ is a field. If furthermore $K\left(X_{Y}\right)$ is isomorphic to the field $K(X)$ of rational functions, then $Y$ is called G3 in $X$. In this note we prove the G3 property for some small codimension subvarieties in projective rational homogeneous spaces.

More precisely, let $G$ be a reductive group, let $P$ be a parabolic subgroup and let $X=G / P$. Denote by $X^{P}(w)$ the Schubert varieties in $X$ (see below for more details). We define (see Definition 2.1) admissible Schubert subvarieties $X^{P}(v)$ in $X^{P}(w)$ and prove

Theorem 0.1. Let $f: Y \rightarrow X$ be a proper morphism and let $X^{P}(v)$ be admissible in $X^{P}(w)$ with $[f(Y)] \cdot\left[X^{P}(v)\right] \neq 0$. Then $f^{-1}\left(X^{P}(w)\right)$ is G3 in $Y$.

Applying this result we obtain formal properties of the inverse image of the diagonal in partial flag varieties: let $X=\mathbb{F}\left(\left(a_{i}\right)_{i \in[1, r]} ; V\right)$ be a variety of partial flags in $V$ (see Section 2.2) and let $H$ be a hyperplane of $V$. Write $\Delta_{H}$ for the diagonal in $\mathbb{F}\left(\left(a_{i}\right)_{i \in[1, r]} ; H\right) \times \mathbb{F}\left(\left(a_{i}\right)_{i \in[1, r]} ; H\right)$ and $\Delta$ for the diagonal in $X \times X$.

Corollary 0.2. Let $f: Y \rightarrow X \times X$ be a proper morphism with $[f(Y)] \cdot\left[\Delta_{H}\right] \neq 0$. Then $f^{-1}(\Delta)$ is $G 3$ in $Y$.

Notation. We work over an algebraically closed field $k$ of characteristic 0 . Let $G$ be a reductive group over $k$ and let $T$ be a maximal torus of $G$. Let $B$ a Borel subgroup containing $T$ and $P$ a parabolic subgroup containing $B$. Denote by $W$ and $W_{P}$ the Weyl groups of $G$ and $P$ and by $W^{P}$ the set of minimal length representatives of $W / W_{P}$. The $B$-orbit closures in $X$ are called Schubert varieties and are indexed by elements $w \in W^{P}$. We write $X^{P}(w)$ for the corresponding Schubert variety.

2000 Mathematics Subject Classification. 14M07,14M17,14B10.

The first author was partially supported by the Spanish Ministerio de Economay Competitividad and by the European Regional Development Fund (ERDF), under the project MTM201125816-C02-(02). 
Given $\alpha$ in the root system of $(G, T)$, denote by $U(\alpha)$ the corresponding unipotent subgroup and by $s_{\alpha}$ the corresponding reflection in $W$.

We write $\Sigma(P)$ for the set of simple roots $\alpha$ such that $U(-\alpha)$ is not contained in $P$. Define $S^{P}(w)$ as the stabiliser in $G$ of $X^{P}(w)$. Then $S^{P}(w)$ is a parabolic subgroup of $G$ containing $B$. Set $\Sigma^{P}(w)=\Sigma\left(S^{P}(w)\right)$. For more details on rational homogeneous spaces and algebraic groups, we refer to [2] and [6] respectively.

\section{Preliminaries Regarding the G3 Property}

1.1. Ring of formal functions. Let $X$ be a scheme and $Y$ be a closed subscheme defined by the sheaf of ideals $I$. Write $Y_{n}=\left(Y, \mathcal{O}_{X} / I^{n}\right)$ for the infinitesimal neighbourhood of order $n$ of $Y$ in $X$. The formal completion of $X$ along $Y$ is the formal scheme $X_{Y}=\lim _{\rightarrow} Y_{n}$.

Definition 1.1. The ring of formal rational functions $K\left(X_{Y}\right)$ of $X$ along $Y$ is the sheaf associated to the presheaf $U \mapsto\left[\mathcal{O}_{X_{Y}}(U)\right]_{0}$ where $[A]_{0}$ denotes the total ring of fractions of $A$.

The ring $K\left(X_{Y}\right)$ is in general not a field. However we have the following result.

Proposition 1.2 (see [1, Corollary 9.10]). Let $X$ be an algebraic variety and let $Y$ be a closed subvariety $X$. Let $u: X^{\prime} \rightarrow X$ be the normalization of $X$. Then $K\left(X_{Y}\right)$ is a field if and only if $u^{-1}(Y)$ is connected.

There is a natural morphism $\alpha_{X, Y}: K(X) \rightarrow K\left(X_{Y}\right)$ (see [1, Page 84]).

Definition 1.3. The subscheme $Y$ is called $G 3$ in $X$ if $\alpha_{X, Y}$ is an isomorphism.

We shall also use the following two results for proving the G3 property.

Proposition 1.4. (see [4] or [1, Corollary 9.13]) Let $f: X^{\prime} \rightarrow X$ be a proper surjective morphism. Suppose $Y$ is G3 in $X$. Then $f^{-1}(Y)$ is G3 in $X^{\prime}$.

Proposition 1.5. (see [1, Proposition 9.23]) Let $f: X^{\prime} \rightarrow X$ be a proper surjective morphism of irreducible varieties and let $Y \subset X$ and $Y^{\prime} \subset X^{\prime}$ be closed subvarieties such that $f\left(Y^{\prime}\right) \subset Y$. Assume that the rings $K\left(X_{Y}\right)$ and $K\left(X_{f^{-1}(Y)}^{\prime}\right)$ are both fields. If $Y^{\prime}$ is $G 3$ in $X^{\prime}$, then $Y$ is $G 3$ in $X$.

1.2. Generating subvarieties. Let $X=G / P$ with $G$ a reductive group and $P$ a parabolic subgroup.

Definition 1.6. Let $Y$ be a closed irreducible subvariety in $X$.

1. Let $y \in Y$ and $\varphi: G \rightarrow Y, g \mapsto g \cdot y$. We define $G_{Y}$ to be the subgroup generated by $\varphi^{-1}(G)$. One easily checks that this definition does not depend on the choice of $y$.

2. The subvariety $Y$ generates $X$ if $G_{Y}=G$

Proposition 1.7 (see [1, Corollary 13.8]). A generating subvariety of $X$ is G3.

Definition 1.8. A Schubert variety $X(t)$ with $t \in W^{P}$ is called minimal generating if $t$ has a reduced expression $s_{\beta_{1}} \cdots s_{\beta_{k}}$ such that the roots $\left(\beta_{i}\right)_{i \in[1, k]}$ are all simple distinct and $\Sigma(P)=\left\{\beta_{1}, \cdots, \beta_{k}\right\}$.

Lemma 1.9. A minimal generating Schubert variety is a generating subvariety. 
Proof. Let $Y=X^{P}(t)$ be a minimal generating Schubert variety with reduced expression $t=s_{\beta_{1}} \cdots s_{\beta_{k}}$ such that the roots $\left(\beta_{i}\right)_{i \in[1, k]}$ are all simple distinct and $\Sigma(P)=\left\{\beta_{1}, \cdots, \beta_{k}\right\}$. We prove by induction on $i$ that $U\left(-\beta_{i}\right) \subset G_{Y}$.

Let $y=t \cdot P \in Y$ and let $\varphi: G \rightarrow X, g \mapsto g \cdot y$. We have $U\left(-\beta_{1}\right) \cdot y=$ $t U\left(-t^{-1}\left(\beta_{1}\right)\right) \cdot P$ and $t^{-1}\left(\beta_{1}\right)=s_{\beta_{k}} \cdots s_{\beta_{2}}\left(-\beta_{1}\right)$. Since all the roots $\left(\beta_{i}\right)_{i \in[1, k]}$ are simple and distinct it follows that $t^{-1}\left(\beta_{1}\right)<0$. Therefore $U\left(-\beta_{1}\right) \subset \varphi^{-1}(Y)$. Since $Y^{\prime}=X\left(s_{\beta_{2}} \cdots s_{\beta_{k}}\right) \subset Y$ it follows that $G_{Y^{\prime}} \subset G_{Y}$ and the result follows by induction.

\section{RESUlts ON EXTENSION OF FORMAL FUnCtions}

2.1. Extension of formal functions for Schubert varieties. In this section we want to extend a connectedness result from [5] to a result on extension of formal functions.

Definition 2.1. Let $w, v \in W^{P}$, we say that $X^{P}(v)$ is admissible in $X^{P}(w)$ if $S^{P}(w) X^{P}(v)=X^{P}(w)$ and $\Sigma^{P}(w) \cap \Sigma^{P}(v)=\emptyset$.

Proposition 2.2 (see [5, Theorem 1.5]). Let $f: Y \rightarrow X=G / P$ a proper morphism with $Y$ irreducible. Let $w, v \in W^{P}$ such that $X^{P}(v)$ is admissible for $X^{P}(w)$. Suppose that $[f(Y)] \cdot\left[X^{P}(v)\right] \neq 0$. Then $f^{-1}\left(X^{P}(w)\right)$ is connected.

We extend this result to a result on the G3 property.

Theorem 2.3. Let $f: Y \rightarrow X=G / P$ a proper morphism. Let $w, v \in W^{P}$ such that $X^{P}(v)$ is admissible in $X^{P}(w)$. Suppose that $[f(Y)] \cdot\left[X^{P}(v)\right] \neq 0$. Then $f^{-1}\left(X^{P}(w)\right)$ is G3 in $Y$.

Proof. Using Propositions 1.2, 1.5 and 2.2, we may assume that $Y$ is normal. Let $Q=S^{P}(v)$ and $Z=\left\{(y, \bar{h}) \in Y \times G / Q \mid f(y) \in h\left(X^{P}(v)\right)\right\}$. Consider the incidence diagram:

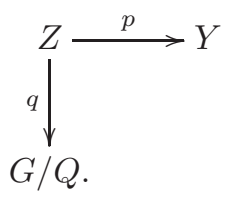

The map $p$ gives $Z$ the structure of a $X^{Q}(u)$-bundle on $X$, where $X^{Q}(u)$ is the closure in $G / Q$ of the $P$-orbit of the Schubert variety $X^{Q}\left(v^{-1}\right)$. Therefore, $p$ is surjective and $Z$ is irreducible. Furthermore, since $[f(Y)] \cdot\left[X^{P}(v)\right] \neq 0$, the map $q$ is also surjective.

Lemma 2.4. Let $X^{Q}(t)$ be a minimal generating Schubert variety in $G / Q$ and let $W=f^{-1}\left(X^{P}(w)\right)$. We have $q^{-1}\left(X^{Q}(t)\right) \subset p^{-1}(W)$.

Proof. The element $t \in W^{Q}$ has a reduced expression $s_{\beta_{1}} \cdots s_{\beta_{k}}$ such that the roots $\left(\beta_{i}\right)_{i \in[1, k]}$ are all simple distinct and $\Sigma(Q)=\left\{\beta_{1}, \cdots, \beta_{k}\right\}$. The Schubert variety $X^{Q}(t)$ is thus the closure of $U\left(-\beta_{1}\right) \cdots U\left(-\beta_{k}\right) \cdot Q$.

We prove $f^{-1}\left(h\left(X^{P}(v)\right) \subset f^{-1}\left(X^{P}(w)\right.\right.$ for $h \in U\left(-\beta_{1}\right) \cdots U\left(-\beta_{k}\right) Q$. It is enough to prove that $U\left(-\beta_{1}\right) \cdots U\left(-\beta_{k}\right) Q \cdot X^{P}(v) \subset X^{P}(w)$. Since $X^{P}(v)$ is admissible, the sets $\Sigma^{P}(w)$ and $\Sigma^{P}(v)=\Sigma(Q)=\left\{\beta_{1}, \cdots, \beta_{k}\right\}$ are disjoint. Therefore, $U\left(-\beta_{i}\right) \subset S^{P}(w)$ for al $i \in[1, k]$. Since $Q$ is the stabiliser of $X^{P}(v)$, we have:

$$
U\left(-\beta_{1}\right) \cdots U\left(-\beta_{k}\right) Q \cdot X^{P}(v)=U\left(-\beta_{1}\right) \cdots U\left(-\beta_{k}\right) \cdot X^{P}(v) \subset X^{P}(w) .
$$


This concludes the proof.

Lemma 1.9 implies that $X^{Q}(t)$ is G3 in $G / Q$ and Proposition 1.4 implies that $q^{-1}\left(X^{Q}(t)\right)$ is G3 in $Z$. Consider $g: \bar{Z} \rightarrow Z$ the normalization of $Z$ and let $W=f^{-1}\left(X^{P}(w)\right)$. By Proposition 2.2 we have that $\bar{W}=g^{-1} p^{-1} f^{-1}\left(X^{P}(w)\right)$ is connected in $\bar{Z}$. Therefore, $K(\bar{Z} \bar{W})=K\left(Z_{p^{-1}(W)}\right)$ is a field. On the other hand, $q^{-1}\left(X^{Q}(t)\right) \subset p^{-1}(W)$ by Lemma 2.4 Applying Proposition 1.5 we get that $p^{-1}(W)$ is $\mathrm{G} 3$ in $Z$ and that $W$ is G3 in $Y$.

2.2. Application to the diagonal of flag varieties. In this section, we prove a G3-Bertini type result for the diagonal of partial flag varieties.

Given a vector space $V$ and a sequence $\left(a_{i}\right)_{i \in[1, r]}$ of positive integers, we write $\mathbb{F}\left(\left(a_{i}\right)_{i \in[1, r]} ; V\right)$ for the variety parametrising partial flags $0 \subset E_{1} \subset \cdots \subset E_{r} \subset V$ where $E_{i}$ is a vector subspace of $V$ of dimension $a_{i}$. If $\operatorname{dim} V=n$, we will also denote this variety by $\mathbb{F}\left(\left(a_{i}\right)_{i \in[1, r]} ; n\right)$. The following result is a variation on a classical trick (see for example [3, Théorème 7.1]).

Let $V$ be an $n$-dimensional vector space. Let $X=\mathbb{F}\left(\left(a_{i}\right)_{i \in[1, r]} ; V\right)$. Let $W=$ $V \oplus V$ and $p_{1}, p_{2}: W \rightarrow V$ be the projections. These projections induce a rational map $p: \mathbb{F}\left(\left(a_{i}\right)_{i \in[1, r]} ; W\right) \rightarrow X \times X$ defined by

$$
\left(E_{1} \subset \cdots \subset E_{r}\right) \mapsto\left(p_{1}\left(E_{1}\right) \subset \cdots \subset p_{1}\left(E_{r}\right), p_{2}\left(E_{1}\right) \subset \cdots \subset p_{2}\left(E_{r}\right)\right) .
$$

Lemma 2.5. The fiber of $p$ is isomorphic to

$$
\prod_{i=1}^{r} \mathrm{GL}\left(a_{i}-a_{i-1}\right)
$$

with $a_{0}=0$.

Proof. Let $(E, F) \in X \times X$ with $E=E_{1} \subset \cdots \subset E_{r} \subset V$ and $F=F_{1} \subset \cdots \subset$ $F_{r} \subset V$. A flag $G=G_{1} \subset \cdots \subset G_{r} \subset W$ is in $p^{-1}(E, F)$ if and only if $p_{1}\left(E_{i}\right)=G_{i}$ and $p_{2}\left(F_{i}\right)=G_{i}$ for all $i$. In that case $G_{i}$ can be seen as the graph in $E_{i} \times F_{i}$ of an isomorphism. Therefore, the whole flag $G$ can be seen as an isomorphism between $E_{r}$ and $F_{r}$ such that the image of $E_{i}$ is $F_{i}$ for all $i<r$. The result follows.

Let $X=\mathbb{F}\left(\left(a_{i}\right)_{i \in[1, r]} ; V\right)$ and let $H$ be a hyperplane of $V$. Write $\Delta_{H}$ for the diagonal in $\mathbb{F}\left(\left(a_{i}\right)_{i \in[1, r]} ; H\right) \times \mathbb{F}\left(\left(a_{i}\right)_{i \in[1, r]} ; H\right)$ and $\Delta$ for the diagonal in $X \times X$. We have an inclusion $\Delta_{H} \subset \Delta$.

Theorem 2.6. Let $f: Y \rightarrow X \times X$ be a proper morphism with $[f(Y)] \cdot\left[\Delta_{H}\right] \neq 0$. Then $f^{-1}(\Delta)$ is $G 3$ in $Y$.

Proof. By Proposition 1.2 and 1.5 we may assume that $Y$ is normal. Let $p$ : $\mathbb{F}\left(\left(a_{i}\right)_{i \in[1, r]} ; W\right) \rightarrow X \times X$ be the rational map defined above and let $\Delta_{W}$ be the diagonal embedding of $V$ in $W=V \oplus V$. Define $\tilde{\Delta}=\mathbb{F}\left(\left(a_{i}\right)_{i \in[1, r]} ; \Delta_{W}\right)$. We have a commutative diagram:

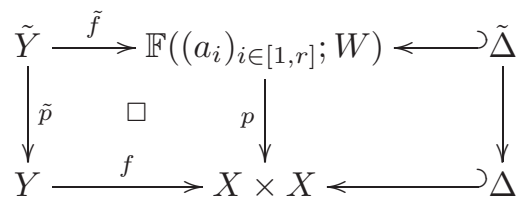


Since $f$ is proper, so is $\tilde{f}$. Note that $\tilde{\Delta}$ is a Schubert variety in $\mathbb{F}\left(\left(a_{i}\right)_{i \in[1, r]} ; W\right)$. Now, let $\tilde{H}$ be a hyperplane of $\Delta_{W}$. Then $\mathbb{F}\left(\left(a_{i}\right)_{i \in[1, r]} ; \tilde{H}\right)$ is an admissible Schubert variety in $\tilde{\Delta}$. On the other hand, $p\left(\mathbb{F}\left(\left(a_{i}\right)_{i \in[1, r]} ; \tilde{H}\right)\right)=\Delta_{H}$ thus $f(Y)$ intersects $\left[\Delta_{H}\right]$. It follows that $\tilde{f}(\tilde{Y})$ intersects $\left.\mathbb{F}\left(\left(a_{i}\right)_{i \in[1, r]} ; H\right)\right]$. Applying Theorem 2.3 $\tilde{f}^{-1}(\tilde{\Delta})$ is $G 3$ in $\tilde{Y}$.

Since $p$ is surjective and $p(\tilde{\Delta})=\Delta$, we get $\tilde{p}\left(\tilde{f}^{-1}(\tilde{\Delta})\right)=f^{-1}(\Delta)$ by base extension. Applying Propositions 1.2 and 1.5 we have that $f^{-1}(\Delta)$ is $G 3$ in $Y$.

Remark 2.7. Using the same technique, similar G3 results on the inverse of the diagonal of isotropic Graßmann varieties can be deduced from connectedness results proved in [5, Theorem 2.2].

\section{REFERENCES}

[1] L. Bădescu, Projective geometry and formal geometry. Monografie Matematyczne Vol. 65, Birkhäuser, 2004.

[2] M. Brion, Lectures on the geometry of flag varieties. Topics in Cohomological Studies of Algebraic Varieties, Trends Math., Birkhuser-Verlag, Basel, 2005, pp. 3385.

[3] O. Debarre, Théorèmes de connexité pour les produits d'espaces projectifs et les grassmanniennes. Amer. J. Math. 118 (1996), no. 6, 1347-1367.

[4] H. Hironaka and H. Matsumura, Formal functions and formal embeddings, J. Math. Soc. Japan 20 (1968), 52-82.

[5] N. Perrin, Small codimension subvarieties in homogeneous spaces. Indag. Math. (N.S.) 20 (2009), no. 4, 557-581.

[6] T.A. Springer, Linear Algebraic Groups. Progress in Mathematics, vol. 9, Birkhuser, Boston, MA, 1981.

Departamento de Álgebra, Facultad de Matemáticas, Plaza de Ciencias, Universidad Complutense de Madrid. 28040 Madrid, Spain

E-mail address: jcaravan@mat.ucm.es

Mathematisches Institut, Heinrich-Heine-Universität, D-40204 Düsseldorf, Germany

E-mail address: perrin@math.uni-duesseldorf.de 